\title{
Accumulation of FIAsH/Lumio Green in active mitochondria can be reversed by $\beta$-mercaptoethanol for specific staining of tetracysteine-tagged proteins
}

\begin{abstract}
Recent advances in the field of small molecule labels for live cell imaging promise to overcome some of the limitations set by the size of fluorescent proteins. We tested the tetracysteine-biarsenical labeling system in live cell fluorescence microscopy of reggie-1/flotillin-2 in $\mathrm{HeLa}$ and N2a cells. In both cell types, the biarsenical staining reagent $\mathrm{FlAsH} /$ Lumio Green accumulated in active mitochondria and led to mitochondrial swelling. This is indicative of toxic side effects caused by arsenic, which should be considered when this labeling system is to be used in live cell imaging. Mitochondrial accumulation of FlAsH/Lumio Green was reversed by addition of low concentrations of thiol-containing reagents during labeling and a subsequent high stringency thiol wash. Both ethanedithiol and $\beta$-mercaptoethanol proved to be effective. We therefore established a staining protocol using $\beta$-mercaptoethanol as thiol binding site competitor resulting in a specific staining of tetracysteine-tagged reggie-1/flotillin-2 of adequate signal to noise ratio, so that the more toxic and inconvenient ethanedithiol could be avoided. Furthermore, we show that staining efficiency was greatly enhanced by introducing a second tetracysteine sequence in tandem.
\end{abstract}

Keywords Tetracysteine $\cdot$ Biarsenical fluorescent ligands · Reggie/flotillin · Live cell imaging ·

Fluorescence microscopy

M.F. Langhorst and S. Genisyuerek contributed equally to this work.

M. F. Langhorst $(\bowtie) \cdot$ S. Genisyuerek · C. A.O. Stuermer Developmental Neurobiology Group, Department of Biology, University of Konstanz, Universitaetsstr. 10, 78457 Konstanz, Germany

E-mail: Matthiaslanghorst $@$ email.de

Tel.: + 49-7531-882128

Fax: $+49-7531-883894$

\section{Introduction}

Investigating the distribution and dynamics of proteins inside living cells by fluorescence microscopy has been greatly simplified by genetically encoded recombinant fusion proteins of green fluorescent protein and its variants (Lippincott-Schwartz et al. 2001). However, fusion to fluorescent proteins, which have a molecular mass of $25-30 \mathrm{kDa}$, can severely impair the function and/or localization especially of small proteins. Smaller labels may overcome some of these problems by reducing steric hindrance (reviewed by Chen and Ting 2005). Labeling of recombinant proteins using the tetracysteine-biarsenical system developed by Tsien and coworkers (Griffin et al. 1998) appears to be particularly promising. It is based on complex formation between a biarsenical variant of fluorescein (fluorescein arsenical helix binder, FlAsH) or resorufin $(\mathrm{ReAsH})$ and a genetically encoded tetracysteine motif of only $6-12$ amino acids. This system cannot only be used for fluorescence detection of proteins in living cells, but also for correlative electron microscopy (Gaietta et al. 2002), fluorophore-assisted light inactivation (FALI) (Marek and Davis 2002; Tour et al. 2003) and pulse-chase experiments (Gaietta et al. 2002). Furthermore, FlAsH and ReAsH are now commercially available as Lumio Green ${ }^{\mathrm{TM}}$ and Lumio $\operatorname{Red}^{\mathrm{TM}}$ from Invitrogen.

We are using the tetracysteine-biarsenical labeling system to investigate the localization and dynamics of reggie-1/flotillin-2. This protein of $47 \mathrm{kDa}$ was discovered in our lab as a protein upregulated in goldfish retinal ganglion cells during axon regeneration after optic nerve injury (Schulte et al. 1997; Lang et al. 1998). Its exact function is still unknown, but it most probably acts as a lipid raft-associated scaffolding protein, defining specialized microdomains for multiprotein complex assembly at cellular membranes (reviewed in Langhorst et al. 2005). 


\section{Materials and methods}

Reagents

Lumio Green and Disperse Blue were purchased from Invitrogen (Karlsruhe, Germany) as part of the "Lumio in cell labeling kit ${ }^{\mathrm{TM}}$ ". Mitotracker Orange and Rhodamine 123 were from Molecular Probes (Leiden, Netherlands). $\quad \beta$-mercaptoethanol (2-ME), ethanedithiol (EDT), formaldehyde and rotenone were from Sigma (Munich, Germany). Oligonucleotides were from Operon Biotechnology (Cologne, Germany). Enzymes for molecular biology were from New England Biolabs (Beverly, USA) or Fermentas (St. Leon-Rot, Germany).

Cloning of reggie-1-tetracysteine expression vectors

To construct expression vectors for reggie-1/flotillin-2 Cterminally fused to one (R1FL-Cys 4 ) or two tetracysteine-sequences (R1FL-(Cys $)_{2}$ ), we excised EGFP from pR1FL-EGFP, a plasmid containing full-length rat reggie-1 cloned into pEGFP-N1 (Clontech) (NeumannGiesen et al. 2004), using BamHI and NotI. By ligation with annealed oligonucleotides (fw: 5'-GATCCATTCCTGAACTGTTGTCCCGGCTGCTGCATGGAGCCTTGA-3'; rv: 5'-GGCCTCAAGGCTCCATGCAG CAGCCGGGACAACAGTTCAGGAATG-3') we then introduced the first tetracysteine sequence coding for FLNCCPGCCMEP*, a tetracysteine sequence with optimized flanking amino acids for particularly high affinity for biarsenical ligands (Martin et al. 2005). To introduce a second tetracysteine sequence, we linearized the resulting plasmid using BamHI, dephosphorylated the ends using calf intestine alkaline phosphatase and ligated phosphorylated, annealed oligonucleotides (fw: Phos-5'-GATCACTCTCTTAACTGCTGCCCGGGGTGTTGTATGGAACCCGTAGTCCTT-3', rv: Phos5'-GATCAAGGACTACGGGTTCCATACAACACCCCGGGCAGCAGTTAAGAGAGT-3') encoding SLNCCPGCCMEP. The use of different codons made colony PCR screening with a specific reverse primer (5'-ATCAAGGACTACGGGTT-3') possible. All constructs were finally sequenced for verification. This strategy proved to be a fast, reliable and versatile way to introduce tetracysteine sequences wherever a restriction site was available or could be introduced.

\section{Cell culture and transfection}

N2a and HeLa cells were cultured in MEM (Invitrogen) supplemented with 100 units $/ \mathrm{ml}$ Penicillin, $50 \mathrm{mg} / \mathrm{ml}$ Streptavidin, $1 \mathrm{mM}$ sodium pyruvate, $2 \mathrm{mM}$ glutamine and $10 \%$ foetal calf serum (all Invitrogen) at $37^{\circ} \mathrm{C}$ and $5 \% \mathrm{CO}_{2}$. Cells were transfected using Lipofectamine 2000 according to the manufacturers instructions. For labeling, transfected cells were plated onto chambered coverslips (Nunc, Rochester, USA) coated with poly-Llysine and laminin (both Sigma) $24 \mathrm{~h}$ after transfection and labeled $48 \mathrm{~h}$ post transfection.

\section{Labeling of HeLa and N2a cells}

Cells were washed with Hepes-buffered salt solution (HBSS) $\left(135 \mathrm{mM} \mathrm{NaCl}, 4.5 \mathrm{mM} \mathrm{KCl}, 1.5 \mathrm{mM} \mathrm{CaCl}_{2}\right.$, $0.5 \mathrm{mM} \mathrm{MgCl} 2,5.6 \mathrm{mM}$ Glucose, $25 \mathrm{mM}$ HEPES, $\mathrm{pH}$ 7.4) and incubated with $0.3-0.5 \mu \mathrm{M} \mathrm{FlAsH} /$ Lumio Green and - if indicated-0.6-4 $\mu \mathrm{M} 2-\mathrm{ME}$ or EDT in HBSS for $1 \mathrm{~h}$. After labeling, cells were thoroughly washed with HBSS and either imaged immediately or incubated with $100 \mu \mathrm{M}$ 2-ME or EDT in HBSS for $30 \mathrm{~min}$ before microscopic analysis.

Fluorescence microscopy

Images were acquired on an Axiovert $200 \mathrm{M}$ using a Plan-Apochromat $63 \times$ objective $(\mathrm{NA}=1.4)$ and an Axiocam MRm operated at full resolution (12 bit, 1,388 $\times 1,040$ pixels; all Carl Zeiss, Jena, Germany). Where indicated, the Apotome system (Carl Zeiss) was used to acquire confocal images by structured illumination. Images were processed using AxioVision 4.4 (Carl Zeiss) and ImageJ (Abramoff et al. 2004).

\section{Results and discussion}

FlAsH/Lumio Green accumulates in active mitochondria

In the absence of thiol reagents during labeling with FlAsH/Lumio Green, we observed in both HeLa and N2a cells a very bright staining of mitochondria, which we verified by co-staining using the mitochondrial marker Mitotracker Orange (Fig. 1a). This bright mitochondrial labeling by FlAsH/Lumio Green prevented the identification of potentially present specific staining of reggie-1/flotillin-2 in cells transfected with R1FL-Cys 4 or R1FL- $\left(\mathrm{Cys}_{4}\right)_{2}$. Furthermore, after FlAsH/Lumio Green labeling we often observed a distinct change of mitochondria morphology towards a round, swollen appearance (Fig. 1b) indicating that the labeling procedure has a toxic effect on mitochondria. Addition of low concentrations $(1-2 \mu \mathrm{M})$ of the dithiol ethanedithiol (EDT) during labeling and a $30 \mathrm{~min}$ wash of the labeled cells with $100 \mu \mathrm{M}$ EDT greatly reduced the mitochondrial accumulation of FlAsH/Lumio Green, but it did not completely prevent the morphological changes. Surprisingly, the monothiol $\beta$-mercaptoethanol (2-ME) proved to be similarly effective in reversing mitochondrial accumulation of $\mathrm{FlAsH} / \mathrm{Lumio}$ Green (Fig. 1c).

To test whether the accumulation of $\mathrm{FlAsH} / \mathrm{Lumio}$ Green in mitochondria is activity-dependent, we inhib- 
Fig. 1 Mitochondrial accumulation of FlAsH/Lumio Green and toxic side effects a Untransfected N2a cells were labeled with $1 \mu \mathrm{M}$ FlAsH/ Lumio Green and Mitotracker Orange in the absence of thiolcontaining reagents for $1 \mathrm{~h}$, washed and analyzed by fluorescence microscopy. FlAsH/Lumio Green clearly accumulated in mitochondria stained by MitoTracker (a confocal slice acquired by structured illumination is shown). b Many cells stained as described above exhibited aberrant mitochondria with a round, swollen appearance $\mathbf{c}$ Untransfected N2a cells were stained with $1 \mu \mathrm{M}$ FlAsH/ Lumio Green $+4 \mu \mathrm{M} 2-\mathrm{ME}$ for $1 \mathrm{~h}$, washed and incubated with $100 \mu \mathrm{M} 2-\mathrm{ME}$ for $30 \mathrm{~min}$. This treatment reversed mitochondrial accumulation of FlAsH/Lumio Green and resulted in a diffuse background staining. d Cells were labeled with $1 \mu \mathrm{M}$ FlAsH/Lumio Green for $1 \mathrm{~h}$, washed and then incubated with $5 \mu \mathrm{M}$ rotenone for $15 \mathrm{~min}$. Inhibition of mitochondrial respiration by rotenone also reversed the mitochondrial accumulation of FlAsH/Lumio Green

fluorescence. All bars, $10 \mu \mathrm{m}$
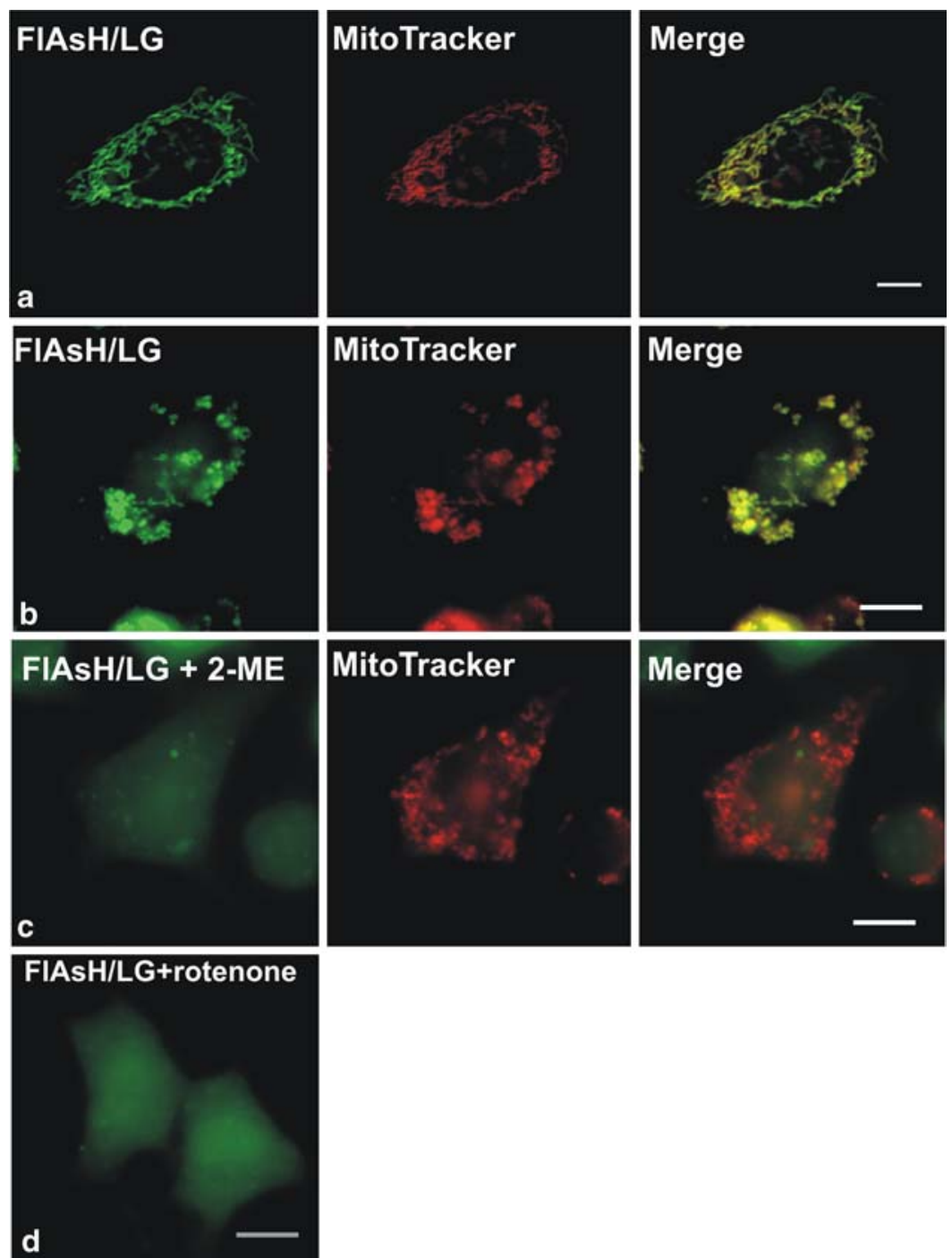

ited mitochondrial respiration by a short incubation with $5 \mu \mathrm{M}$ Rotenone (Lindahl and Oberg 1960). This treatment abolished labeling of the mitochondria by FlAsH/Lumio Green (Fig. 1d), suggesting that accumulation and/or fluorescence of FlAsH/Lumio Green in mitochondria is somehow linked to mitochondrial activity. Uptake of Rhodamine 123 into mitochondria, which only occurs in active mitochondria and which could readily be inhibited by rotenone treatment, was, however, not affected by the 2-ME concentrations necessary to reduce mitochondrial staining by $\mathrm{FlAsH} / \mathrm{Lu}-$ mio Green (data not shown), suggesting that the thiol reagents act only by competing with cellular thiol binding sites in mitochondria and do not impair mitochondrial function.

Several other treatments were reported to reduce background staining while using the tetracysteine-biarsenical system. Disperse Blue is a hydrophobic dye which suppresses unspecific staining by blocking hydrophobic binding sites (Griffin et al. 2000) and is part of the labeling kit sold by Invitrogen. Addition of pyruvate was also suggested as a means of reducing background staining (Griffin et al. 2000). However, both reagents had no discernible effect on mitochondrial accumulation of FlAsH/Lumio Green.

The FlAsH/Lumio Green-induced morphological changes of the mitochondria might reflect toxic effects of arsenic, which is known to disrupt mitochondrial function by generating reactive oxygen species and perturbing the GSH redox system (Dalton 2002). These side effects might severely compromise the value of this labeling system for live cell imaging. In our experiments, including EDT or 2-ME in the labeling procedure was mandatory, as a high stringency wash with EDT or 2ME proved to be the only efficient way to reverse the accumulation of FlAsH/Lumio Green in mitochondria. Furthermore, addition of low concentrations of thiols during labeling reduced morphological changes of the mitochondria induced by FlAsH/Lumio Green at least to a certain extent, probably by reversing the toxic effect of arsenic on the GSH redox system (Zmuda and Friedenson 1983). Still, the toxic side effects of the biarsen- 

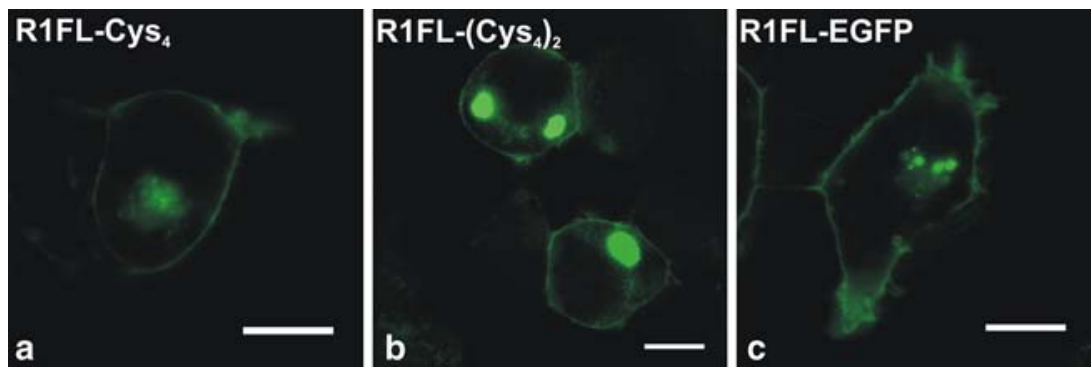

Fig. 2 R1FL-Cys 4 / R1FL-(Cys $\left.)_{2}\right)_{2}$ labeling in N2a cells using $\beta$ mercaptoethanol: $\mathrm{N} 2 \mathrm{a}$ cells were transiently transfected with $\mathrm{R}_{1 F L-C y s}$ (a), R1FL-(Cys $)_{2}$ (b) or R1FL-EGFP (c). R1FL$\mathrm{Cys}_{4} / \mathrm{R} 1 \mathrm{FL}-\left(\mathrm{Cys}_{4}\right)_{2}$ expressing cells were stained with $0.3 \mu \mathrm{M}$

ical labeling reagent on mitochondria have to be considered when choosing a labeling system, especially for long-term live cell imaging.

Specific staining of tetracysteine-tagged reggie in the presence of $\beta$-mercaptoethanol

So far, all publications using the tetracysteine-biarsenical labeling system utilized EDT to suppress unspecific binding of the biarsenical fluorescent staining reagent to cellular thiols. EDT is highly toxic, not readily soluble in physiological salt solutions and even after extensive washing, the stench emanating from EDT-treated samples is almost unbearable. Encouraged by our observation that the mitochondrial accumulation of FlAsH/Lumio Green was efficiently inhibited by addition of 2-ME, we tested whether we could establish conditions for specific labeling of tetracysteine-tagged reggie-1/flotillin-2 in the presence of 2-ME only. Incubation of cells transiently transfected with R1FL-Cys 4 with $0.3-0.5 \mu \mathrm{M} \mathrm{FlAsH} /$ Lumio Green in the presence of an 4-6x excess of 2-ME for $1 \mathrm{~h}$, followed by a 30 min wash with $100 \mu \mathrm{M} 2-\mathrm{ME}$ resulted in a clear, reggie-1/flotillin-2-specific staining of the plasma membrane and intracellular vesicles (Fig. 2a), very much comparable to the localization observed in R1FL-EGFP expressing cells (Fig. 2c). This clearly demonstrates that specific labeling using the tetracysteine-biarsenical system can be achieved by replacing EDT with the less toxic, easier to handle 2-ME. This suggests that the bivalency of EDT is not indispensable and that an excess of a monothiol like 2-ME can also efficiently suppress unspecific binding of the biarsenical fluorescent ligand to cellular thiols.

The staining efficiency using R1FL-Cys 4 with a single tetracysteine-sequence was relatively low, but introducing a second tetracysteine sequence in tandem improved staining efficiency significantly (Fig. 2b). Although all constructs were expressed at similar levels (data not shown), the staining efficiency could be more than doubled by a second tetracysteine sequence. We therefore suggest testing different constructs with variable numbers and localizations of tetracysteine
FlAsH/Lumio Green $+1.2 \mu \mathrm{M}$ 2-ME with a subsequent wash with $100 \mu \mathrm{M} 2-\mathrm{ME}$ and analyzed by fluorescence microscopy. All bars, $10 \mu \mathrm{m}$

sequences, as not only localization of the tag seems to be crucial (N-terminal tagging of reggie-1/flotillin-2 for example results in a mislocalized fusion protein most probably because myristoylation of Gly2 is inhibited), but staining efficiency and intensity might also be influenced. A previous report on FlAsH-labeling in yeast cells stated that introducing a tandem tetracysteine sequence increased the photostability of the labeling (Andresen et al. 2004).

The signal to noise ratio obtained by our staining procedure was adequate for many applications, but in comparison with R1FL-EGFP expressing cells (Fig. 2c) clearly inferior. The lower signal to noise ratio was caused by a diffuse FlAsH/Lumio Green staining of the whole cell, which is most probably due to binding of the biarsenical reagent to intracellular cysteine-rich proteins as previously described (Stroffekova et al. 2001). This background staining was further reduced by increasing the 2-ME concentration during the high stringency wash, but this led to a severe loss of cells due to detachment from the coverslip, as similarly observed for EDT-treatment.

The fact that the FlAsH/Lumio Green label was subject to photobleaching was another problem. FlAsH/ Lumio Green is a biarsenical variant of fluorescein, which is known to photobleach rapidly (Song et al. 1995). The recent description of a rational design of new fluorescein variants with improved properties (Urano et al. 2005) might allow the development of more photostable variants, which would greatly facilitate live cell imaging of FlAsH/Lumio Green labeled proteins.

In summary we have demonstrated that specific staining of tetracysteine-tagged proteins using biarsenical fluorescent ligands is possible without ethanedithiol, which we replaced by $\beta$-mercaptoethanol, which is less toxic and more convenient to handle. A thiol-reagent proved to be mandatory in the labeling procedure to reverse the accumulation of FlAsH/Lumio Green in active mitochondria. The mitochondrial accumulation of the biarsenical labeling reagent was accompanied by mitochondrial swelling, which could only partly reversed by thiol-containing reagents. These toxic side effects might limit the usefulness of the tetracysteine-biarsenical labeling system for live cell imaging. 
Acknowledgements We thank Gonzalo Solis and Alexander Reuter for their help with cloning of the R1-Cys 4 -expression vectors. This work was supported by grants from the Deutsche Forschungsgemeinschaft DFG (SFB-TR11), the Ministerium Forschung, Wissenschaft und Kunst Baden-Württemberg (TSE program) and the Fonds der Chemischen Industrie.

\section{References}

Abramoff MD, Magelhaes PJ, Ram SJ (2004) Image processing with image. J Biophotonics Int 11:36-42

Andresen M, Schmitz-Salue R, Jakobs S (2004) Short tetracysteine tags to beta-tubulin demonstrate the significance of small labels for live cell imaging. Mol Biol Cell 15:5616-5622

Chen I, Ting AY (2005) Site-specific labeling of proteins with small molecules in live cells. Curr Opin Biotechnol 16:35-40

Dalton WS (2002) Targeting the mitochondria: an exciting new approach to myeloma therapy. Commentary re: Bahlis NJ et al. (2002) Feasibility and correlates of arsenic trioxide combined with ascorbic acid-mediated depletion of intracellular glutathione for the treatment of relapsed/refractory multiple myeloma. Clin Cancer Res 8:3658-3668. Clin Cancer Res 8:3643-3645

Gaietta G, Deerinck TJ, Adams SR, Bouwer J, Tour O, Laird DW, Sosinsky GE, Tsien RY, Ellisman MH (2002) Multicolor and electron microscopic imaging of connexin trafficking. Science 296:503-507

Griffin BA, Adams SR, Jones J, Tsien RY (2000) Fluorescent labeling of recombinant proteins in living cells with FlAsH. Methods Enzymol 327:565-578

Griffin BA, Adams SR, Tsien RY (1998) Specific covalent labeling of recombinant protein molecules inside live cells. Science 281:269-272

Lang DM, Lommel S, Jung M, Ankerhold R, Petrausch B, Laessing U, Wiechers MF, Plattner H, Stuermer CA (1998) Identification of reggie-1 and reggie- 2 as plasmamembrane-associated proteins which cocluster with activated GPI-anchored cell adhesion molecules in non-caveolar micropatches in neurons. J Neurobiol 37: 502-523
Langhorst MF, Reuter A, Stuermer CA (2005) Scaffolding microdomains and beyond: the function of reggie/flotillin proteins. Cell Mol Life Sci 62:2228-2240

Lindahl PE, Oberg KE (1960) Mechanism of the physiological action of rotenone. Nature 187:784

Lippincott-Schwartz J, Snapp E, Kenworthy A (2001) Studying protein dynamics in living cells. Nat Rev Mol Cell Biol 2: 444-456

Marek KW, Davis GW (2002) Transgenically encoded protein photoinactivation (FlAsH-FALI): acute inactivation of synaptotagmin I. Neuron 36:805-813

Martin BR, Giepmans BN, Adams SR, Tsien RY (2005) Mammalian cell-based optimization of the biarsenical-binding tetracysteine motif for improved fluorescence and affinity. Nat Biotechnol 23(10):1308-1314

Neumann-Giesen C, Falkenbach B, Beicht P, Claasen S, Luers G, Stuermer CA, Herzog V, Tikkanen R (2004) Membrane and raft association of reggie-1/flotillin-2: role of myristoylation, palmitoylation and oligomerization and induction of filopodia by overexpression. Biochem J 378:509-518

Schulte T, Paschke KA, Laessing U, Lottspeich F, Stuermer CA (1997) Reggie-1 and reggie-2, two cell surface proteins expressed by retinal ganglion cells during axon regeneration. Development 124:577-87

Song L, Hennink EJ, Young IT, Tanke HJ (1995) Photobleaching kinetics of fluorescein in quantitative fluorescence microscopy. Biophys J 68:2588-600

Stroffekova K, Proenza C, Beam KG (2001) The protein-labeling reagent FLASH-EDT2 binds not only to CCXXCC motifs but also non-specifically to endogenous cysteine-rich proteins. Pflugers Arch 442:859-66

Tour O, Meijer RM, Zacharias DA, Adams SR, Tsien RY (2003) Genetically targeted chromophore-assisted light inactivation. Nat Biotechnol 21:1505-1508

Urano Y, Kamiya M, Kanda K, Ueno T, Hirose K, Nagano T (2005) Evolution of fluorescein as a platform for finely tunable fluorescence probes. J Am Chem Soc 127:4888-4894

Zmuda J, Friedenson B (1983) Changes in intracellular glutathione levels in stimulated and unstimulated lymphocytes in the presence of 2-mercaptoethanol or cysteine. J Immunol 130:362-364 
